\title{
About possibility of bistable dynamics in lasers with single-mode cavities
}

\author{
Vladimir Shuvayev, ${ }^{1}$ Vinod Menon, ${ }^{1,2}$ Alexander Lisyansky, ${ }^{1}$ and Lev Deych ${ }^{1, *}$ \\ ${ }^{1}$ Physics Department, Queens College of CUNY, Flushing, New York 11367, USA \\ ${ }^{2}$ Hybrid Photonics, LLC, 104 Addison Lane, Greenvale, New York 11548, USA \\ ${ }^{*}$ Corresponding author: lev.deych@qc.cuny.edu
}

Received October 3, 2012; revised October 29, 2012; accepted October 30, 2012; posted October 31, 2012 (Doc. ID 177413); published December 6, 2012

\begin{abstract}
A previously unknown mechanism of bistable behavior in lasers with single-mode cavities is proposed and analyzed. It is shown that if losses in a cavity exhibit nonmonotonic dependence on frequency, the equation for stationary lasing frequencies can have multiple solutions even in single-mode cavities. In such a case, a system can generate one of several lasing outputs characterized by different frequencies and intensities. All these potential lasing states are stable at the same pumping level, and the choice between them is determined by initial conditions. The latter can be, in principle, controlled by seeding pulses. This mechanism does not depend on such nonlinear effects responsible for most known types of bistability as saturable absorption or cross saturation. An example of a cavity structure, in which such a mechanism can be realized, is presented. Standard lasing equations fail to describe dynamical behavior of such systems; therefore a generalized approach treating dynamic of lasing frequency and intensity on equal footing is developed. (c) 2012 Optical Society of America
\end{abstract}

OCIS codes: $\quad 190.1450,140.3430,140.3570,130.4815$.

\section{INTRODUCTION}

Bistability is one of the most important manifestations of nonlinear effects in various dynamic systems and it has been the subject of intensive research. While bistability is characteristic for nonlinear systems of various kinds, optical bistability has attracted special attention during the last three decades (see, for instance, [1-4] and references therein) because of its importance for optical information processing. A special case of optical bistable systems are optical amplifiers and lasers whose output demonstrates bistable behavior as a function of the pumping power [므료. Most known sources of bistability rely on intensity-dependent changes of real (dispersive bistability [9-11]) or imaginary (absorptive bistability $[2,12-19])$ parts of the refractive index of the medium. Typically, bistability in these situations occurs when a function representing the output intensity, $I_{\text {out }}$, versus the input power, $P_{\text {in }}$, acquires a shape characterized by the existence of two critical values of $P_{\text {in }}$ at which the derivative $d I_{\text {out }} / d P_{\text {in }}$ changes sign passing through infinity. Function $I_{\text {out }}\left(P_{\text {in }}\right)$ in the interval between these values becomes multivalued with three (or more) possible values of $I_{\text {out }}$ for each $P_{\text {in }}$. Usually only two of these solutions are stable, resulting in a familiar hysteresis behavior. In lasers, bistability is also known to take place due to other mechanisms such as mode competition [20] and frequency-dependent feedback $[21,22]$. Bistability described in [20] occurs when two stable lasing modes coexist at the same pumping level, but mode competition makes a two-mode operation unstable. In $[21,22]$ the bistability is created by combining a laser with an external frequency-selective feedback, which again results in a multivalued form of function $I_{\text {out }}\left(P_{\text {in }}\right)$ or, as in the case described in [15], in a multivalued dependence of lasing frequency versus input power.

In this paper, we propose and theoretically study a mechanism of multistable behavior that differs principally from previously known mechanisms. It is realized in lasing structures utilizing single-mode cavities with frequencydependent losses exhibiting multiple oscillations over a frequency interval containing the cavity mode. Due to this oscillatory behavior of the losses, an equation for stationary lasing frequencies in such structures can have multiple solutions. Each of these solutions yields a distinct lasing output with its own frequency, intensity, and the threshold. Some of these different lasing regimes can be stable at the same value of the pumping power. The choice between these simultaneously stable lasing states is determined by the initial conditions, which can be, in principle, controlled by seeding pulses. In order to emphasize that this type of multistable behavior does not depend on mode competition between cavity modes (it takes place in the single-mode cavities) and/or external nonlinear elements such as, for instance, saturable absorbers, we will call it singlemode intrinsic multistability (SMIM).

It should be emphasized that the existence of several stationary frequencies and the single-mode nature of lasing oscillations do not constitute a contradiction in terms. Indeed, in the multimode regime, each lasing mode is characterized by its own field function and frequency, which can be traced to distinct individual modes of the "cold" cavity (there might exist certain exceptions in low- $Q$ cavities, when a lasing mode is a combination of several modes of the "cold" cavity $[\underline{23}, \underline{24}]$, but this situation has no bearing on the case under study in this paper). In this paper we consider a situation in which it is presumed that only one cavity mode is within the gain spectrum of the active medium, which, however, can yield several alternative values for the stationary lasing frequency. Unlike bistable solutions in truly multimode lasers, these alternative frequencies cannot coexist so that no effects of cross saturation and mode competition usually accompanying multimode lasing occur in the situation under consideration. 
It should be noted that the existence of multiple lasing frequencies in single-mode lasers, albeit arising for completely different reasons, has been also proposed in recent papers $[25,26]$ concerned with systems with spatially nonuniform gain. According to $[25,26]$, in systems with nonuniform gain, in addition to the regular lasing mode originating from the modes of the "cold" cavity, there might exist a surface mode propagating along the boundary between the regions with different gain values. This additional mode is characterized by its own stationary frequency and a much higher lasing threshold. Despite formal similarity (several lasing frequencies arising from a single cavity mode), the phenomenon considered in this paper is drastically different from the one studied in $[25,26]$. Indeed, in the case here, the lasing output at any of the multiple frequencies corresponds to the same spatial "mode" with field distributions at all lasing frequencies being very similar to each other and to the mode of the "cold" cavity. As a result, the lasing thresholds for different frequencies are not drastically different from each other.

The existence of multiple frequency solutions in a singlemode stationary regime makes the description of relaxation kinetics less trivial than in the standard situations. Usually, the relaxation to the stationary lasing state is described via slow-changing amplitude approximation, which involves timedependent equations for intensity and the phase of the field containing only the first-order time derivatives of the respective quantities. Neglecting the time derivatives of the phase of higher orders is tantamount to assuming that the nonstationary contribution to the instantaneous value of the lasing frequency does not have its own dynamics and follows adiabatically the intensity [27]. In the situation under consideration in this paper, such an approach is insufficient, and the second-order time derivative of the phase plays an important role and has to be taken into account. In the single-mode regime this can be achieved by rewriting the phase equation as a first-order differential equation for frequency and analyzing the coupled intensity-frequency dynamics considering both parameters as equal partners in establishing the final stationary state of the laser.

\section{NORMAL FREQUENCIES AND MODES OF THE STRUCTURE}

The schematic of the structure under consideration in the paper is shown in Fig. 1. This structure consists of two waveguides filled with an active medium and mirrored at their left ends (the length of the active portion of the each waveguide is $L)$. The waveguides are coupled to each other via a disk or ring resonator of radius $R$. We will show that this structure can be described as an effective Fabry-Perot cavity of length $2 L$ with frequency-dependent losses.

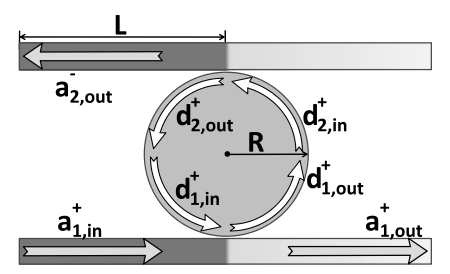

Fig. 1. Schematic of a structure considered in the paper: two waveguides filled with a gain medium (shaded area) are coupled to each other via a passive ring resonator.
For simplicity we shall assume that the structure is constructed of single-mode symmetrical waveguides supporting a $\mathrm{TE}_{00}$ mode characterized by zero cutoff frequency. The field in the waveguides can be presented in the following form:

$$
\begin{aligned}
& E_{1}=\left\{\begin{array}{ll}
A^{(+)} e^{i k^{(1)} z}+A^{(-)} e^{-i k^{(1)} z}, & z<L \\
T^{(1)} e^{i k^{(1)} z}, & z>L
\end{array},\right. \\
& E_{2}=\left\{\begin{array}{ll}
B^{(+)} e^{i k^{(2)} z}+B^{(-)} e^{-i k^{(2)} z}, & z<L \\
T^{(2)} e^{i k^{(2)} z}, & z>L
\end{array},\right.
\end{aligned}
$$

where $k_{1,2}(\omega)$ are the propagation constants of a given waveguide mode in the bottom and top waveguides, respectively, and $z$ is the coordinate along the waveguides counted from their mirrored ends. Equation (1) is complemented by relations between amplitudes $A^{(+)}, B^{(+)}$and $A^{(-)}, B^{(-)}$,

$$
A^{(+)}=r A^{(-)}, \quad B^{(+)}=r B^{(-)}
$$

where $r$ is a reflection coefficient at the mirrored ends of the waveguides. We will assume that the coupling region between the waveguides and the ring is much smaller than all other lengths in the system so that its size can be neglected. The coupling between the resonator and the waveguides will be described using the phenomenological approach introduced in [28]. If an incident signal propagates in a first (bottom) waveguide in a positive (to the right) direction, it couples to the counterclockwise propagating mode of the resonator, which, in its turn, couples to a wave propagating to the left in the second (top) waveguide. This situation is described by introducing amplitudes $a_{1, \text { in }}^{+}$and $d_{1, \text { in }}^{+}$of the field incident on the bottom coupling region in the waveguide and the resonator, respectively, as well as amplitudes $a_{1, \text { out }}^{+}$and $d_{1, \text { out }}^{+}$describing fields coming out of the bottom coupling region in the waveguide and the resonator, respectively. These amplitudes are related to each other via matrix relations

$$
\left(\begin{array}{l}
a_{1, \text { out }}^{+} \\
d_{1, \text { out }}^{+}
\end{array}\right)=\left(\begin{array}{cc}
p & i \kappa \\
-i \kappa & p
\end{array}\right)\left(\begin{array}{l}
a_{1, \text { in }}^{+} \\
d_{1, \text { in }}^{+}
\end{array}\right),
$$

where $p$ and $\kappa$ are coefficients that characterize this coupling region. The energy conservation requires that these coefficients obey relation $|p|^{2}+|\kappa|^{2}=1$. At the top coupling region, one introduces amplitudes $d_{2, \text { in }}^{+}$and $d_{2, \text { out }}^{+}$for the field incident on the coupler in the resonator, and amplitude $a_{2}^{-}$out for the outgoing field in the waveguide. Since the direction of incoming and outgoing fields at the second coupler is reversed compared to the first coupling region, the respective amplitudes are related via a Hermitian conjugated matrix

$$
\left(\begin{array}{c}
a_{2, \text { out }}^{-} \\
d_{2, \text { out }}^{+}
\end{array}\right)=\left(\begin{array}{cc}
p^{*} & i \kappa^{*} \\
-i \kappa^{*} & p^{*}
\end{array}\right)\left(\begin{array}{c}
0 \\
d_{2, \text { in }}^{+}
\end{array}\right) .
$$

Equations (3) and (4) must be complemented by relations between amplitudes of the field in the resonator taking into account their internal dynamics. These relations can be written as

$$
\begin{aligned}
& d_{2, \text { in }}^{+}=\alpha e^{-i \Phi / 2} d_{1, \text { out }}^{+}, \\
& d_{1, \text { in }}^{+}=\alpha e^{-i \Phi / 2} d_{2, \text { out }}^{+},
\end{aligned}
$$


where $\Phi=n_{r} \omega L_{r} / c$ is a single round-trip change of the phase of a wave of frequency $\omega$ in a resonator with the refractive index $n_{r}$ and the length $L_{r}$ and the coefficient $\alpha$ introduces losses in the couplers combined with propagating losses in parts of the resonator between the two coupling regions. We assume that the couplers are identical so that the complete round-trip losses in the resonator are described by $\alpha^{2}$.

The mirrors at the ends of the waveguides complicate the situation by producing a field in the top waveguide propagating to the right. This field excites a clockwise propagating mode in the resonator, which couples to the field in the bottom waveguide propagating to the left. Thus, Eqs. (3)-(5), in the case under consideration, must be complemented by equations describing this additional coupling process. To this end, we introduce new amplitudes $a_{2 \text {,in }}^{+}, a_{2, \text { out }}^{+}, a_{1, \text { out }}^{-}$for the incident and outgoing field in the waveguides, and respective amplitudes $d_{2, \text { in }}^{-}, d_{2, \text { out }}^{-}, d_{1, \text { in }}^{-}, d_{1, \text { out }}^{-}$for the field in the resonator. The upper index “-” indicates clockwise propagating modes of the resonator, while all other indices here and in expressions for waveguide amplitudes are the same as previously introduced. Incident and outgoing amplitudes are related to each other by equations analogous to Eqs. (3) and (4), where, however, the former corresponds to the coupling region in the top waveguide, and the later to the bottom waveguide. Relations between the amplitudes of the resonator field at different coupling regions is now given by expressions

$$
d_{1, \text { in }}^{-}=\alpha e^{i \Phi / 2} d_{2, \text { out }}^{-}, d_{2, \text { in }}^{-}=\alpha e^{i \Phi / 2} d_{1, \text { out }}^{-},
$$

where the sign change in the exponential terms explicitly takes into account the clockwise nature of the resonator's mode.

Using Eqs. (3)-()), one can relate amplitudes of the left-propagating waves in the top waveguide and the rightpropagating wave in the bottom waveguide:

$$
a_{2, \text { out }}^{-}=\frac{\alpha|\kappa|^{2} e^{-i \Phi / 2}}{1-\alpha^{2}|p|^{2} e^{-i \Phi}} a_{1, \mathrm{in}}^{+} .
$$

Using Eq. (1) one can rewrite the relation given by Eq. (7) in terms of the respective field amplitudes at $z=0$ :

$$
B^{(-)} e^{-i k^{(2)} L}=\frac{\alpha|\kappa|^{2} e^{-i \Phi / 2}}{1-\alpha^{2}|p|^{2} e^{-i \Phi}} A^{(+)} e^{i k^{(1)} L}
$$

In a similar manner, we can relate fields coupled to the clockwise mode of the resonator:

$$
A^{(-)} e^{-i k^{(2)} L}=\frac{\alpha|\kappa|^{2} e^{i \Phi / 2}}{1-\alpha^{2}|p|^{2} e^{i \Phi}} B^{(+)} e^{i k^{(1)} L} .
$$

Multiplying Eq. (ㅁ) by Eq. (ㅁ) and taking into account the relation between the amplitudes given by Eq. (2), one obtains a secular equation for the propagating constants of the normal modes in the structure under consideration:

$$
e^{-2 i\left(k^{(1)}+k^{(2)}\right) L}=\frac{\alpha^{2} r^{2}|\kappa|^{4}}{\left.\left.|1-\alpha| p\right|^{2} e^{i \Phi}\right|^{2}} .
$$

Since the right-hand side of Eq. (10) is a real-valued quantity, it is obvious that solutions to this equation are necessarily complex-valued, reflecting an inherently open nature of the structure under consideration. Presenting the propagation constants $k_{m}^{(1,2)}$ in the form $k_{m}^{(1,2)}=q_{m}^{(1,2)}+i \delta_{m}^{(1,2)}$, we find that the real part of the propagation constants obeys equation

$$
\left(q_{m}^{(1)}+q_{m}^{(2)}\right) L=\pi m .
$$

Equation (11) shows that even though the resonator provides coupling between the waveguides, it does not affect the spectral characteristics of the resulting cavity. Its role is to control radiative losses of the cavity by determining the imaginary part of the propagation constant:

$$
\left(\delta_{m}^{(1)}+\delta_{m}^{(2)}\right) L=-\frac{1}{2} \ln S(\omega)
$$

where

$$
S=\frac{\alpha^{2} r^{2}|\kappa|^{4}}{1+\alpha^{4}|p|^{4}-2 \alpha^{2}|p|^{2} \cos \left(2 \pi \frac{\omega}{\omega_{r}}\right)},
$$

with $\omega_{r}=(2 \pi c) /\left(n_{r} L_{r}\right)$. The resonator function $S$ obeys inequality $0<S(\omega)<1$, ensuring thereby the positive sign of $\delta_{m}^{(1,2)}$.

The system of Eqs. (8) and (9) also determines the ratio of the field amplitudes in the waveguides

$$
\frac{B_{m}^{(-)}}{A_{m}^{(-)}}=(-1)^{m} e^{i \psi}
$$

where

$$
\psi=\Phi / 2-\tan ^{-1} \frac{|p|^{2} \alpha^{2} \sin \Phi}{1-|p|^{2} \alpha^{2} \cos \Phi} .
$$

Normal modes of the cavity are usually used as a basis to represent an arbitrary field distribution in it. However, the open nature of the structure under consideration in this paper, manifested through complex modal propagation constants, makes the standard expansion procedure impossible. Indeed, the normal modes described above are not eigenfunctions of a Hermitian operator, and as such, do not have to be orthogonal to each other in the usual sense of Hermitian inner product. The problem of introducing normal modes in open systems has been discussed extensively in the past (see, for instance, recent review in [29]) and is usually solved through introduction of a dual set of adjoint modes forming a biorthogonal system [30]. In this paper we will use an approach introduced in the context of laser physics in [31], which utilizes so-called "constant-flux modes" (CFMs). The basis of CFMs has been used to formulate so-called steady-state ab initio laser theory generalizing earlier theories to the case of lasers with complex cavities $[23,24,31-33]$.

Adopting this approach to the situation under consideration, we introduce the following set of adjoint functions, $U_{m}^{(1,2)}(z)$ and $\bar{U}_{m}^{(1,2)}(z)$ defined in the bottom and top waveguides, respectively, as

$$
\begin{aligned}
& U_{m}^{(1,2)}(z)=r e^{i k_{m}^{(1,2)} z}+e^{-i k_{m}^{(1,2)} z}, \\
& \bar{U}_{m}^{(1,2)}(z)=r e^{i\left[k_{m}^{(1,2)}\right]^{*} z}+e^{-i\left[k_{m}^{(1,2)}\right]^{*} z} .
\end{aligned}
$$

We have not included into the definition of these modal functions the phase $\psi$ defined in Eq. (15), as it is more convenient 
to include this phase in the respective modal amplitudes, when using Eq. (16) to construct the total field in the waveguides. It is important that CFMs are defined with boundary conditions at infinity formulated in terms of the real-valued generic spectral parameter $\omega$, which later becomes identified with the lasing frequency, rather than in terms of complexvalued eigenfrequencies [23,31-33]. This circumstance distinguishes CFM from so called quasi- or Gamow modes [29] diverging at infinity, and enables one to introduce a biorthogonality condition involving integration over the space occupied by cavity only. Taking into account that Eq. (11) for the modal propagation constants indicates that the coupled waveguides behave as an effective cavity of total length $2 L$, one can conjecture that the biorthogonality condition in the particular case considered here can be written down as

$$
\int_{0}^{L} U_{m}^{(1)}(z)\left[\bar{U}_{n}^{(1)}(z)\right]^{*} \mathrm{~d} z+\int_{L}^{2 L} U_{m}^{(2)}(z)\left[\bar{U}_{n}^{(2)}(z)\right]^{*} \mathrm{~d} z=N_{m} \delta_{m n},
$$

where normalization factor $N_{m}$ is complex-valued because of the open nature of the effective resonator.

An important consequence of using a generic spectral parameter to define behavior of the CFMs outside of the cavity is that these modes, as well as their eigenfrequencies, become $\omega$-dependent. In our case this is manifested via $\omega$-dependence of the imaginary parts of the propagating constants and of the interwaveguide phase, $\psi$. This dependence plays an important role in determining lasing properties of the structure under consideration.

\section{DERIVATION OF LASING EQUATIONS}

The system of the modes presented in Eq. (16) can be used as a basis for expansion of the electric field in the waveguides in the presence of the gain medium and to derive equations for the respective modal amplitudes. Since this derivation deviates from the textbook examples [27,30], we sketch it briefly in this section of the paper.

In the presence of an active medium, the field in each waveguide satisfies equations

$$
\frac{n_{w}^{2}}{c^{2}} \frac{\partial^{2} E^{(1,2)}}{\partial t^{2}}-\frac{\partial^{2} E^{(1,2)}}{\partial z^{2}}=-\frac{4 \pi}{c^{2}} \frac{\partial^{2} P^{(1,2)}}{\partial t^{2}},
$$

where $n_{w}$ is the refractive index of the waveguides and $P^{(1,2)}$ is the polarization of the gain medium in each waveguide. Within the framework of the standard semiclassical laser theory, this field is presented in the form of the linear combination of the cavity modes defined in Eq. (16) with time-dependent amplitudes, which are separated into a part oscillating at yet unknown lasing frequencies $\Omega_{m}$ and slowly changing amplitude $A_{m}^{(1,2)}(t)=\left|A_{m}^{(1,2)}(t)\right| e^{i \varphi_{m}(t)}$ :

$$
E^{(1,2)}(z, t)=\frac{1}{2} \sum_{m}\left[\left|A_{m}^{(1,2)}(t)\right| e^{-i \Omega_{m} t-i \varphi_{m}^{(1,2)}} U_{m}^{(1,2)}(z)+\text { c.c. }\right]
$$

In the traditional semiclassical laser theory only the absolute value and the phase of the amplitude $A_{m}$ are allowed to have its own relaxation dynamics described by the respective time derivative, while the lasing frequencies $\Omega_{m}$ are assumed to follow the amplitude adiabatically. Hence, the time derivative of the frequency is neglected. We will see, however, that in the situation under consideration in this work, the relaxation toward stationary lasing states can only be described correctly if one treats both the amplitude and the lasing frequency on equal footing taking into account terms with frequency time derivatives $d \Omega_{m} / d t$.

In order to connect this approach with a more conventional treatment based on time-dependent phase, one can combine $\Omega_{m} t$ term in Eq. (19) with the phase term into a form

$$
\varphi_{m}(t)=\varphi_{0}+\int_{0}^{t} \Omega_{m}(\tau) \mathrm{d} \tau
$$

which is similar to quasi-classical expression for the phase of the wavefunction of a quantum particle in a spatially smooth potential. In this case, when introducing the slow changing amplitude approximation, one should retain time derivatives of the phase up to the second order. It is more convenient, however, to assign the time dependence directly to $\Omega_{m}$ as it is done in Eq. (19).

The idea of time-dependent lasing frequency reflects the presence of fast and slow time scales in the system, which allows for introducing frequencies dependent of the "slow" time via Fourier transform with respect to the "fast" time. Physically, time dependence of lasing frequencies reflects the fact that in the course of establishing stationary lasing output, an "average" frequency of an initial spontaneously emitted spectrally broad signal slowly evolves toward its stationary value. In usual situations, when each mode is characterized by a single stationary value of the lasing frequency, the dynamic of this process is not important. If, however, a single-mode can evolve to one of several stationary frequencies, the frequency dynamics becomes as important as that of intensity. We show below that the possibility of multiple stationary frequencies in single-mode cavities can indeed be realized in the system described in this paper.

Since we consider here a single-mode cavity, we can simplify the derivation of the lasing equations by retaining only one term in Eq. (19). Allowing explicit dependence of the lasing frequencies upon time and introducing standard rotating waves and slow changing amplitude approximations, we obtain

$$
\begin{aligned}
& {\left[-2 i \frac{d\left|A_{m}^{(1,2)}\right|}{d t}+\left|A_{m}^{(1,2)}\right|\left(\omega_{m}^{(1,2)}-\frac{d \varphi_{m}^{(1,2)}}{d t}-t \frac{d \Omega_{m}}{d t}-\Omega_{m}\right)\right] U_{m}^{(1,2)}(z)} \\
& \quad=4 \pi \Omega_{m} P_{m}^{(1,2)}(z, t)
\end{aligned}
$$

where $P_{m}^{(1,2)}(z, t)$ is a positive frequency component of the polarization of the active medium in the respective waveguide. We also introduced eigenfrequencies, $\omega_{m}^{(1,2)}=c k_{m}^{(1,2)} / n_{w}$ of the waveguide modes characterized by propagation constants $k_{m}^{(1,2)}$. One should note, however, that Eq. (10), which determines propagation constants of the normal modes of the coupled waveguide-resonator system, only specifies their sum $k_{m}^{(1)}+k_{m}^{(2)}$, leaving their individual values undefined. While in the stationary state characterized by the frequency $\Omega_{m}$, it is obvious that $k_{m}^{(1)}=k_{m}^{(2)}=\Omega_{m} n_{w} / c$, this is not so when the system is still in the process of relaxation. During this process the phase difference between the field in the top and bottom waveguides, expressed by Eqs. (14) and (15), changes due to the time dependence of $\Omega$. The time derivatives 
of the respective phases, which appear in Eq. (20), contribute to the apparent frequencies of the field in their respective waveguides. These apparent frequencies must be equal to each other, resulting in the following condition imposed on the propagation constants:

$$
\omega_{m}^{(1)}-\frac{d \varphi_{m}^{(1)}}{d t}=\omega_{m}^{(2)}-\frac{d \varphi_{m}^{(2)}}{d t} .
$$

This condition, in combination with Eqs. (11) and (14), allows one to define values of propagation constants $k_{m}^{(1,2)}$ and respective eigenfrequencies $\omega_{m}^{(1,2)}$ :

$$
\omega_{m}^{(1,2)}=\nu_{m}-i \xi_{m} \pm \frac{1}{2} \frac{d \psi}{d t}
$$

where

$$
\nu_{m}=m \omega_{B} ; \quad \xi_{m}=\frac{\omega_{B}}{2 \pi} \ln S\left(\Omega_{m}\right),
$$

with

$$
\omega_{B}=\frac{c \pi}{2 n_{w} L},
$$

being the fundamental frequency of the effective lasing cavity.

Now one can eliminate the modal functions from Eq. (20) using the orthogonality condition of Eq. (17) and taking into account that $\left|A_{m}^{(1)}\right|=\left|A_{m}^{(2)}\right|$, according to Eq. (14). The resulting equation for the amplitude and frequency of the lasing mode takes the form

$$
\begin{gathered}
-2 i \frac{d|A|}{d t}+|A|\left(\nu+i \xi-\frac{1}{2} \frac{d \psi}{d t}-t \frac{d \Omega}{d t}-\Omega\right) \\
=\frac{4 \pi \Omega}{N} \int_{0}^{2 L} P^{+}(z, t)[\bar{U}(z)]^{*} \mathrm{~d} z,
\end{gathered}
$$

where $N$ is the normalization constant defined in Eq. (17), $P^{+}=P^{(1)}+P^{(2)}$, and we abridged the notations by dropping superficial mode index $m$.

Using a standard two-level model of the gain medium and calculating the polarization term in the constant population approximation [27] we can rewrite Eq. (25), separating its real and imaginary parts, as

$$
\begin{aligned}
& \frac{d \Omega}{d t}\left(t+\pi \frac{1+W(\Omega)}{2 \omega_{r}}\right)+\Omega \\
& \quad=\nu+P \Omega g(\Omega)\left[\frac{\omega_{0}-\Omega}{\gamma_{\perp}} F_{1}(I, \Omega)-F_{2}(I, \Omega)\right],
\end{aligned}
$$

where

$$
W(\Omega)=\frac{2|p|^{2} \alpha^{2}\left(\cos \Phi-|p|^{2} \alpha^{2}\right)}{1-2|p|^{2} \alpha^{2} \cos \Phi+|p|^{4} \alpha^{4}},
$$

originates from the time derivative of the phase difference $\psi$ expressed in terms of the time derivative of frequency $\Omega$, and

$$
\frac{d I}{d t}=\frac{\omega_{B}}{2 \pi} I \ln S(\Omega)+P \Omega g(\Omega) I\left[F_{1}(I, \Omega)+\frac{\omega_{0}-\Omega}{\gamma_{\perp}} F_{2}(I, \Omega)\right],
$$

where $I$ is dimensionless intensity defined as

$$
I=|A|^{2} \frac{|\varsigma|^{2}}{2 \hbar^{2} \gamma_{\|} \gamma_{\perp}}
$$

via dipole matrix element of the lasing transition $\varsigma$ and the relaxation rate of the population inversion $\gamma_{\|}$. In Eqs. (26) and (28), we also introduced the effective pumping rate $P$ and dimensionless gain Lorentzian

$$
g(\Omega)=\frac{\gamma_{\perp}^{2}}{\gamma_{\perp}^{2}+\left(\omega_{0}-\Omega\right)^{2}},
$$

where $\gamma_{\perp}$ and $\omega_{0}$ are the dephasing rate and the central frequency of the atomic transition responsible for gain. Functions $F_{1}$ and $F_{2}$ in Eqs. (26) and (28) are defined as

$$
\begin{aligned}
& F_{1}(I, \Omega)=\operatorname{Re}\left(\frac{1}{N} \int_{0}^{2 L} \frac{U(z) \bar{U}^{*}(z)}{1+R} \mathrm{~d} z\right) \\
& F_{2}(I, \Omega)=\operatorname{Im}\left(\frac{1}{N} \int_{0}^{2 L} \frac{U(z) \bar{U}^{*}(z)}{1+R} \mathrm{~d} z\right)
\end{aligned}
$$

where the saturation parameter $R$

$$
R=I|U(z)|^{2} g(\Omega),
$$

takes into account saturation of the population inversion. Presence of function $F_{2}(I, \Omega)$ in Eq. (26) is the consequence of the openness of the effective lasing cavity in the structure under consideration. Since in the closed cavities $U(z) \equiv \bar{U}(z)$, the integral in Eq. (31) becomes real valued, rendering $F_{2}=0$. We will see in the following sections of the paper that this term plays an important role in determining the lasing properties of the system.

\section{BISTABLE LASING}

\section{A. Stationary Regime}

We begin our study of the laser emission described by Eq. (26) by considering the stationary regime, when both frequency and intensity time derivatives turn to zero. Lasing $(I \neq 0)$ stationary states are found as solutions of the following system of equations for the stationary frequency and intensity:

$$
\begin{aligned}
& \Omega-\nu=P \Omega g(\Omega)\left[\frac{\omega_{0}-\Omega}{\gamma_{\perp}} F_{1}(I, \Omega)-F_{2}(I, \Omega)\right] \\
& \ln S(\Omega)=-2 \pi \frac{P \Omega g(\Omega)}{\omega_{B}}\left[F_{1}(I, \Omega)+\frac{\omega_{0}-\Omega}{\gamma_{\perp}} F_{2}(I, \Omega)\right] .
\end{aligned}
$$

When $F_{2}$ vanishes (nearly closed cavities), one can immediately reduce Eq. (33) to a closed-form equation for the stationary lasing frequencies:

$$
\ln S(\Omega)=\frac{2 \pi \gamma_{\perp}}{\omega_{B}} \frac{\Omega-\nu}{\Omega-\omega_{0}},
$$


which is independent of pumping level as long as it exceeds the lasing threshold. The latter is obtained by setting $I=0$ in the second of Eq. (33):

$$
P_{\mathrm{th}}=-\frac{\omega_{B}}{2 \pi \Omega g(\Omega)} \ln S(\Omega)
$$

In open cavities, similar to the one considered in this paper, function $F_{2}$ does not vanish, making the analysis more complicated. Equation (34) in this case defines the lasing frequency only at the lasing threshold, which can be seen considering that $F_{1}(0, \Omega)=1 ; F_{2}(0, \Omega)=0$. Above the threshold, the frequency, which now depends on $P$, must be found together with intensity from full system (33).

Despite the fact that Eq. (34) is valid only at $P=P_{\text {th }}$ it provides an important insight into the properties of the lasing in this system. Under certain conditions, which can be controlled by the design of the structure, this equation allows for multiple solutions. Multiplicity of the stationary frequencies creates conditions for possible multistable behavior since the system in the single-mode regime can only laze at one of them at any given time. This mechanism of multistability significantly differs from all other known mechanisms: it does not rely on cross-saturation effects in multimode operations and does not require any additional nonlinear elements such as saturable absorbers [2, $12-\underline{19}]$. As was mentioned in the introduction, we shall call this phenomenon SMIM to emphasize its independence of the mode competition and external nonlinear elements.

The left-hand side of Eq. (34) is always negative, while its right-hand side changes from negative infinity to zero between $\omega_{0}$ and $\nu$, thus guaranteeing the existence of at least one stationary frequency in this interval. The possibility of multiple solutions derives from periodicity of $S(\omega)$, which oscillates between its minimum value

$$
S^{(\min )}=\left(\frac{\kappa^{2} \alpha r}{1+\alpha^{2} p^{2}}\right)^{2},
$$

occurring at

$$
\Omega_{n}^{(\min )}=\left(n-\frac{1}{2}\right) \omega_{r}, \quad n=1,2,3 \ldots
$$

and its maximum value

$$
S^{(\max )}=\left(\frac{\kappa^{2} \alpha r}{1-\alpha^{2} p^{2}}\right)^{2}
$$

at

$$
\Omega_{n}^{(\max )}=n \omega_{r}, \quad n=1,2,3 \ldots
$$

It is necessary, while not sufficient, for the existence of multiple solutions that $S(\Omega)$ undergoes several oscillations when $\Omega$ moves through the gain spectrum. For this to happen, the fundamental frequency of the resonator must obey inequality $\omega_{r}<\gamma_{\perp}$, which imposes limits for the minimum size of the resonator: $L_{r} \gg(2 \pi c) /\left(n_{r} \gamma_{\perp}\right)$. In addition, the function at the right-hand side of Eq. (34) must bend downward fast enough.
As a guide in the design of structures with multiple lasing frequencies, one can use the following set of inequalities ensuring the existence of at least three solutions:

$$
\begin{aligned}
& \left|\ln S_{\min }\right|<\frac{2 \pi \gamma_{\perp}}{\omega_{B}}\left|\frac{\Omega_{n}^{(\min )}-\nu}{\Omega_{n}^{(\min )}-\omega_{0}}\right| \\
& \left|\ln S_{\max }\right|>\frac{2 \pi \gamma_{\perp}}{\omega_{B}}\left|\frac{\Omega_{n}^{(\max )}-\nu}{\Omega_{n}^{(\max )}-\omega_{0}}\right| .
\end{aligned}
$$

These inequalities can be satisfied by manipulating the size of the effective cavity determined by parameter $L$.

An example of the situation, in which Eq. (34) has three solutions, is shown in Fig. 2. The calculations were carried out for the following values of parameters $r=0.8,|p|^{2}=$ $0.51, \alpha^{2}=0.9, \nu=1.0002 \omega_{0}, \gamma_{\perp}=0.001 \omega_{0}$, and $n_{w} L=5.24 \lambda_{0}$; $n_{r} L_{r}=2 \times 10^{4} \lambda_{0}$, where $\lambda_{0}$ is the vacuum wavelength at atomic frequency $\omega_{0}$. This choice of the parameters corresponds to a very lossy cavity with the $Q$-factor oscillating between minimum value of $Q_{\min } \approx 50$ and maximum value of $Q_{\max } \approx 175$. This choice of parameters was made to demonstrate that the phenomenon under consideration does not require cavities with extremely high $Q$-factors; thus its observation will not be hindered by parasitic nonlinear effects, which might occur in high- $Q$ cavities.

It should be understood that each of the solutions shown in Fig. $\underline{2}$ exist at different pumping rates corresponding to their respective lasing threshold. Enumerating the frequencies such that $\Omega_{1}>\Omega_{2}>\Omega_{3}$, one can show that the respective thresholds obey inequalities $P_{\text {th }}^{(1)}<P_{\text {th }}^{(2)}<P_{\text {th }}^{(3)}$. The system starts lasing when $P$ slightly exceeds the lowest threshold $P_{\text {th }}^{(1)}$ at a frequency equal to $\Omega_{1}$, while two other solutions are not relevant at this point as they correspond to nonlasing states. With increasing pumping, the actual frequency of the lasing mode deviates from $\Omega_{1}$ and is determined together with respective intensity by the full system of Eq. (33). When the pumping reaches value $P=P_{\text {th }}^{(2)}$, the possibility of the second lasing state with frequency $\Omega=\Omega_{2}$ emerges. Further increase of pumping rate results in the frequencies of the both potential lasing states to deviate from their respective threshold values. Finally, at $P=P_{\text {th }}^{(3)}$ the third potential lasing solution with $\Omega=\Omega_{3}$ becomes possible.

Solving the complete system of equations Eq. (33), one can find stationary frequencies and respective intensities for

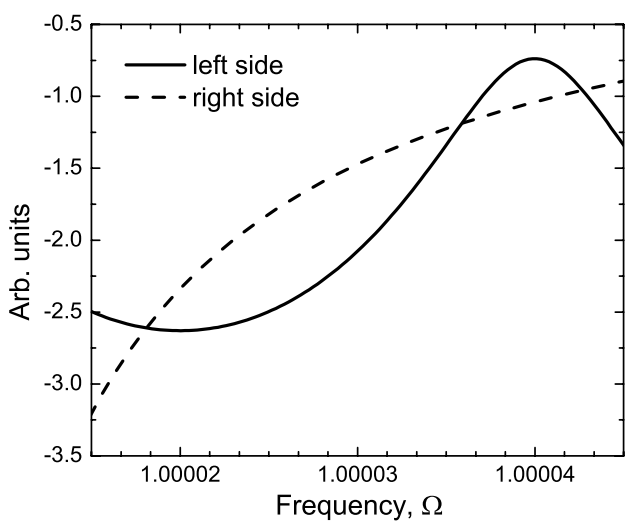

Fig. 2. Left-and right-hand sides of Eq. (34) as functions of frequency normalized by the frequency of atomic transition, illustrating the existence of three stationary frequencies. 
various values of pumping. Figure $\underline{3}$ shows the results of numerical solution of this system, demonstrating dependence of possible lasing frequencies upon pumping emerging whenever $P$ exceeds respective thresholds. The bottom panel of this figure shows pumping dependence of the lasing intensity for each of the possible stationary frequencies. One can see that when $P>P_{\text {th }}^{(3)}$, three stationary solutions with different values of lasing frequency and intensity coexist, signifying a possibility of multistable behavior.

The results presented in Fig. 3 reflect several features of the structure under consideration, distinguishing it from other multistable lasing systems. First of all, the intensity, as a function of pumping, does not show a standard hysteresislike behavior typical for bistable systems. Instead, at a certain value of pumping rate, the two stationary frequencies together with respective intensities coalesce and disappear when the pumping is further increased. This phenomenon is a direct consequence of the openness of the system under consideration, and is sensitive to the parameters of the structure. Another important feature of this system is that the intensity, which normally determines radiative properties of lasers, is subordinate to the frequency. It is the behavior of the latter as a function of the pumping rate that determines the number and properties of the lasing states in the system.

\section{B. Relaxation Dynamics and Stability Analysis}

The actual realization of multistable behavior depends, of course, on the stability of the found stationary solutions. Equation (26) for the frequency contains explicit dependence on time, making the standard linear stability analysis impossible. Therefore, one is forced to rely on direct numerical simulation of time-dependent Eqs. (26) and (28) for various values of the pumping and initial conditions. The results of the simulations are presented in Fig. 4. Immediately above the lowest of the threshold values $P_{\text {th }}^{(2)}>P>P_{\text {th }}^{(1)}$, the solution with zero intensity becomes unstable, and a single stable state, corresponding to the frequency $\Omega_{1}$ and its respective intensity, emerges (see Fig. 3). As this behavior is trivial, it is not presented in Fig. 4. When the pumping rate exceeds the second threshold, $P_{\mathrm{th}}^{(3)}>P>P_{\mathrm{th}}^{(2)}$, a new situation is observed: while the $\Omega_{1}$ solution remains stable, the nonlasing solution also regains its stability. The behavior of the system is determined

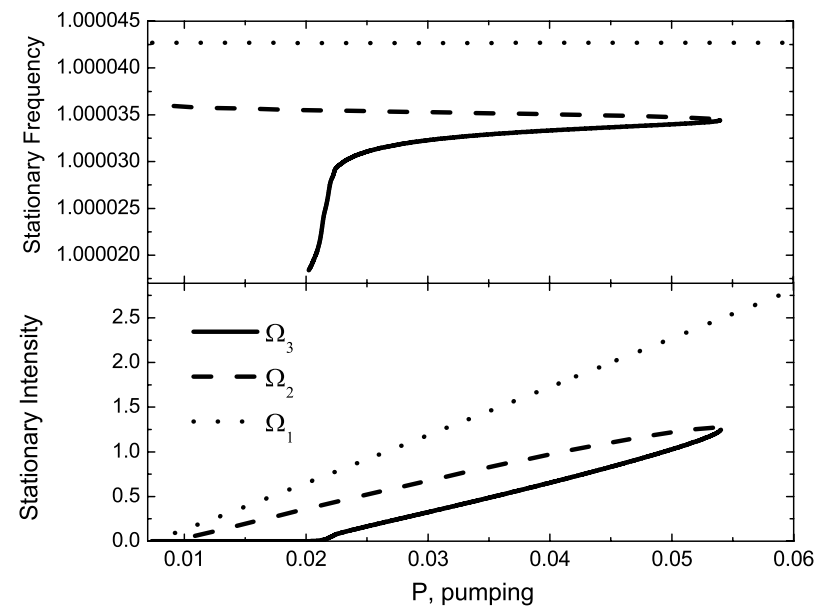

Fig. 3. Dependence of the stationary frequencies and respective lasing intensities on the strength of pumping.

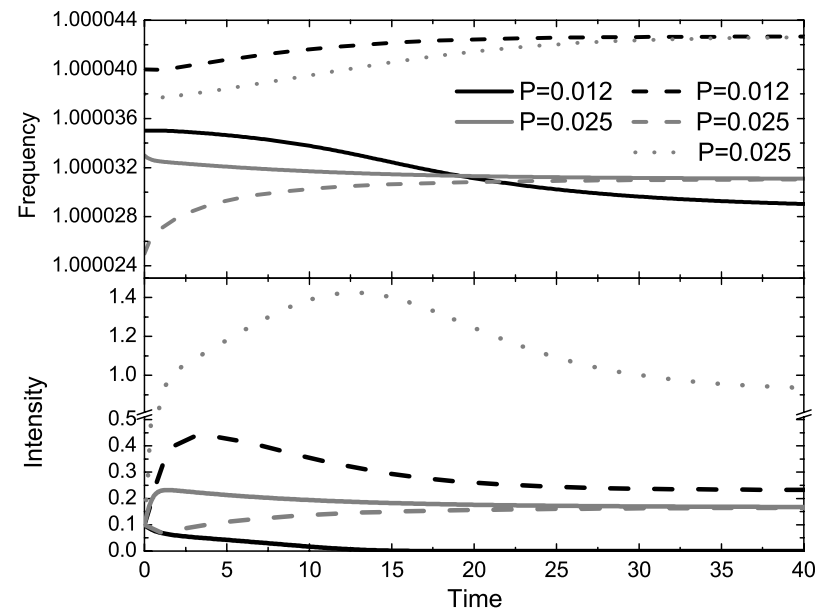

Fig. 4. Temporal evolution of the emission intensity and frequency toward their stationary values for various pumping rates and initial conditions.

mostly by the initial value of the frequency, $\Omega_{\text {in }}$ : if $\Omega_{\text {in }}$ is below $\Omega_{2}$ at the same pumping rate, the system evolves toward zerointensity solution (solid black curves in Fig. 4), but if $\Omega_{\text {in }}>\Omega_{2}$, the system relaxes toward the $\Omega_{1}$ lasing state (black dashed curves in Fig. 4). The $\Omega_{2}$ solution remains unstable for all values of $P$, but plays an important role of separating the regions of initial conditions evolving to different stationary states. The principal role of the frequency as opposed to the intensity in this system is also seen from the fact that it is the initial value of frequency, which, in most cases, determines the final point of the evolution. While we observed situations when extremely small initial values of the intensity would prevent the system from relaxing to the lasing state, it would occur only when the dynamics of frequency would bring it at some point below $\Omega_{2}$ even if the initial frequency is above this value. Coexistence of stable lasing and nonlasing states at the same pumping level is fairly typical for systems with saturable absorbers [ $\underline{4}, \underline{34}]$, and was also reported in [21].

With further increase of the pumping rate $P_{\text {th }}^{(3)}<P<P_{c r}$, the nonlasing solution loses its stability, but the $\Omega_{3}$ lasing solution becomes stable. The original $\Omega_{1}$ solution still retains its stability so that in this range of pumping one has two simultaneously stable lasing states, which differ from each other by their intensities and frequencies. The actual realization of one or the other of these states again depends on the position of the initial frequency with respect to the unstable $\Omega_{2}$ solution. This situation is shown by gray curves in Fig. $\underline{4}$, where we plot curves obtained for three different initial conditions, two of which (solid and dashed) relax to the $\Omega_{3}$ solution, and one (dotted) converges to the $\Omega_{1}$ solution. Eventually, for the chosen values of the systems' parameters, there exists another threshold value of pumping, $P_{c r}$, at which $\Omega_{3}=\Omega_{2}$ and above which both these solutions disappear. Thus, for $P>P_{c r}$, one is left again with a single lasing state.

\section{CONCLUSION}

We demonstrated in this paper that there exist special types of structures in which a previously unknown mechanism of bistability can be realized. This mechanism exists in a single-mode lasing regime, does not require additional nonlinear elements, and depends on linear properties of the lasing structure. The 
system investigated shows several different types of bistability such as the coexistence of simultaneously stable lasing and zero-intensity states or of two lasing states with different intensities and frequencies. Control over the actual lasing state is provided by initial conditions, which can be prepared with the help of seeding pulses. However, to better understand switching dynamics of this system and its stability with respect to spontaneous emission noise, additional studies should be conducted. An interesting feature of this structure is the existence of a bifurcation point, at which two of stationary states collapse in one, and disappear. This bifurcation can result in a sudden change of the lasing intensity and frequency provoked by a very small change of the pumping intensity. Thus, the system studied in this paper demonstrates several types of bistable behavior based on a linear bistability mechanism, and can have potential applications in all-optical logical devices and optical memory.

Consideration in this paper was conducted in the singlemode approximation. This approximation remains valid for all the range of considered pumping intensities, if the threshold for two mode lasing exceeds the highest level of pumping required for observation of the effects considered in the paper. To establish an accurate criterion for the stability of the obtained results with respect to emergence of two-mode lasing, one needs to develop a multimode lasing theory for the system under consideration, which is outside of the scope of this paper. One can, however, note that by making the free spectral range of the effective cavity exceed the width of the gain line of the active medium, one almost guarantees the absence of the two-mode lasing in the system. To achieve this, the active region of the waveguides forming the structure must be much smaller than the circumference of the disk or ring resonator. Practically this can be achieved by defining the active area of the waveguides by using periodic grating on its surface as a mirror.

\section{ACKNOWLEDGMENTS}

The authors gratefully acknowledge financial support of AFOSR under Contract No. FA9550-10-C-0055.

\section{REFERENCES}

1. C. M. Bowden, M. Ciftan, and H. R. Robl, Optical Bistability (Plenum, 1981).

2. P. Mandel, Theoretical Problems in Cavity Nonlinear Optics (Cambridge University, 1997).

3. K. Otsuka, Nonlinear Dynamics in Optical Complex Systems (Kluwer, 1999).

4. N. N. Rosanov, Spatial Hysteresis and Optical Patterns (Springer, 2002).

5. H. Kawaguchi, Bistabilities and Nonlinearities in Laser Diodes (Artech House, 1994).

6. H. M. Gibbs, Optical Bistability: Controlling Light with Light (Academic, 1985).

7. A. T. Rosenberger, L. A. Orozco, and H. J. Kimble, "Observation of absorptive bistability with 2-level atoms in a ring cavity," Phys. Rev. A 28, 2569-2572 (1983).

8. A. Joshi and M. Xiao, "Optical multistability in three-level atoms inside an optical ring cavity," Phys. Rev. Lett. 91, 143904 (2003)

9. A. J. Vanwonderen and L. G. Suttorp, "Dispersive optical bistability in a nonideal Fabry-Perot cavity. 1. Stability analysis of the Maxwell-Bloch equations," Z. Phys. B 83, 135-142 (1991).
10. T. Vivero, J. M. Rivas-Moscoso, A. P. Gonzalez-Marcos, and J. A. Martin-Pereda, "Dispersive optical bistability in quantum wells with logarithmic gain," IEEE J. Quantum Electron. 46, 1184-1190 (2010).

11. H. A. Batarfi, "Dispersive switching in bistable models," J. Nonlinear Opt. Phys. 17, 265-273 (2008).

12. S. T. Dembinski, A. Kossakowski, L. A. Lugiato, and P. Mandel, "Semi-classical and quantum-theory of bistability in lasers containing saturable absorbers 2," Phys. Rev. A 18, 1145-1151 (1978).

13. L. A. Lugiato, P. Mandel, S. T. Dembinski, and A. Kossakowski, "Semi-classical and quantum theories of bistability in lasers containing saturable absorbers," Phys. Rev. A 18, 238-254 (1978).

14. H. Kawaguchi, "Optical bistability and chaos in a semiconductorlaser with a saturable absorber," Appl. Phys. Lett. 45, 1264-1266 (1984).

15. E. Arimondo, D. Dangoisse, C. Gabbanini, E. Menchi, and F. Papoff, "Dynamic behavior of bistability in a laser with a saturable absorber," J. Opt. Soc. Am. B 4, 892-899 (1987).

16. J. M. Oh and D. H. Lee, "Strong optical bistability in a simple L-band tunable erbium-doped fiber ring laser," IEEE J. Quantum Electron. 40, 374-377 (2004).

17. L. Guidoni, R. Mannella, V. Isaia, P. Verkerk, and E. Arimondo, "Stochastic resonance in a laser with saturable absorber," Nuovo Cimento D 17, 803-810 (1995).

18. S. Djabi, H. Boudoukha, and M. Djabi, "Optical bistability in a laser containing a saturable absorber," Ann. Phys. 32, 63-65 (2007).

19. C. Masoller, M. Oria, and R. Vilaseca, "Modeling a semiconductor laser with an intracavity atomic absorber," Phys. Rev. A 80, 013830 (2009).

20. S. Ishii and T. Baba, "Bistable lasing in twin microdisk photonic molecules," Appl. Phys. Lett. 87, 181102 (2005).

21. A. V. Naumenko, N. A. Loiko, and T. Ackemann, "Analysis of bistability conditions between lasing and nonlasing states for a vertical-cavity surface-emitting laser with frequency-selective optical feedback using an envelope approximation," Phys. Rev. A 76, 023802 (2007).

22. B. Farias, T. P. de Silans, M. Chevrollier, and M. Oria, "Frequency bistability of a semiconductor laser under a frequency-dependent feedback," Phys. Rev. Lett. 94, 173902 (2005).

23. H. E. Tureci, A. D. Stone, L. Ge, S. Rotter, and R. J. Tandy, "Ab initio self-consistent laser theory and random lasers," Nonlinearity 22, C1-C18 (2009).

24. L. Ge, Y. D. Chong, and A. D. Stone, "Steady-state ab initio laser theory: generalizations and analytic results," Phys. Rev. A 82, 063824 (2010).

25. L. Ge, Y. D. Chong, S. Rotter, H. E. Tureci, and A. D. Stone, "Unconventional modes in lasers with spatially varying gain and loss," Phys. Rev. A 84, 023820 (2011).

26. M. Liertzer, L. Ge, A. Cerjan, A. D. Stone, H. E. Tureci, and S Rotter, "Pump-induced exceptional points in lasers," Phys. Rev. Lett. 108, 173901 (2012).

27. M. Sargent, M. O. Scully, and W. E. Lamb, Laser Physics (Addison-Wesley, 1974).

28. A. Yariv, "Universal relations for coupling of optical power between microresonators and dielectric waveguides," Electron. Lett. 36, 321-322 (2000).

29. O. Zaitsev and L. Deych, "Diagrammatic semiclassical laser theory," Phys. Rev. A 81, 023822 (2010).

30. A. E. Siegman, Lasers (University Science, 1986).

31. H. E. Tureci, A. D. Stone, and B. Collier, "Self-consistent multimode lasing theory for complex or random lasing media," Phys. Rev. A 74, 043822 (2006).

32. H. E. Tureci, A. D. Stone, and L. Ge, "Theory of the spatial structure of nonlinear lasing modes," Phys. Rev. A 76, 013813 (2007).

33. H. E. Tureci, L. Ge, S. Rotter, and A. D. Stone, "Strong interactions in multimode random lasers," Science 320, 643-646 (2008).

34. K. Staliunas and V. J. Sánchez Morcillo, Transverse Patterns in Nonlinear Optical Resonators (Springer, 2003). 